Cipango Cahiers d'études japonaises

Hors-série | 2008

Autour du Genji monogatari

\title{
De nouveaux mondes
}

Appariement et recontextualisation de poèmes tirés de romans dans le cadre du Monogatari nihyakuban utaawase

New Worlds - matching and recontextualization of poems selected from novels within the Monogatari nihyakuban utaawase

\section{Michel Vieillard-Baron}

\section{OpenEdition}

\section{Journals}

Édition électronique

URL : https://journals.openedition.org/cipango/596

DOI : 10.4000/cipango.596

ISSN : 2260-7706

Éditeur

INALCO

Édition imprimée

Date de publication : 1 janvier 2008

Pagination : 111-132

ISBN : 978-2-85831-170-5

ISSN : $1164-5857$

Référence électronique

Michel Vieillard-Baron, « DE Nouveaux Mondes », Cipango [En ligne], Hors-série | 2008, mis en ligne le 11 juin 2012, consulté le 30 juin 2021. URL : http://journals.openedition.org/cipango/596 ; DOI : https://doi.org/10.4000/cipango.596

Ce document a été généré automatiquement le 30 juin 2021.

\section{(c) (1) (9)}

Cipango est mis à disposition selon les termes de la Licence Creative Commons Attribution - Pas d'Utilisation Commerciale 4.0 International. 


\title{
De nouveaux mondes
}

\author{
Appariement et recontextualisation de poèmes tirés de romans dans le \\ cadre du Monogatari nihyakuban utaawase \\ New Worlds - matching and recontextualization of poems selected from novels \\ within the Monogatari nihyakuban utaawase
}

Michel Vieillard-Baron

Pour ce qui est des poèmes, le Genji et le Sagoromo sont sans pareils.

Meigetsuki, Tenpuku 1 (1233), $3^{e}$ mois, $20^{e}$ jour

Le texte auquel nous allons nous intéresser ici est une œuvre étonnante. Il s'agit d'un corpus de quatre cents poèmes, waka, extraits de romans, monogatari, que le fameux Fujiwara no Teika (1162-1241) a appariés à la manière des concours de poèmes, utaawase, sans toutefois les départager ni écrire d'attendus de jugements (hanji 判詞) ${ }^{1}$. Les quatre cents waka sont répartis en deux concours; le premier, intitulé Hyakuban utaawase «Concours de poèmes en cent manches » ou Genji Sagoromo utaawase 源氏狭衣 歌合 "Concours de poèmes du Genji et du Sagoromo », contient cent poèmes du Genji monogatari appariés à cent pièces du Sagoromo monogatari «Le roman de Bel Habit », un autre grand roman de l'époque de Heian ${ }^{2}$. Le second, intitulé Go hyakuban utaawase 後百 番歌合 《Second concours de poèmes en cent manches » ou Shüi hyakuban utaawase 拾 遺百番歌合 “Concours en cent manches de poèmes glanés parmi les délaissés", comporte cent nouveaux poèmes du Genji appariés à cent pièces empruntées à dix romans, qui, à l'exception du Yoru no nezame (Réveillés dans la nuit), sont aujourd'hui perdus. Dans le premier concours, les poèmes sont rangés par thèmes à l'instar d'une anthologie : amour (43 manches), séparation (4 manches), voyage (6 manches), deuil (15 manches), sujets divers (32 manches); dans le second, les poèmes sont classés non plus par thèmes mais par romans. La date de compilation de ces deux concours n'a pu être définie avec certitude. Une seule chose est sûre, ils ont été compilés avant 1206, année de la mort du commanditaire, Fujiwara (Kujō) no Yoshitsune 藤原（九条）良経 (1169-1206), poète de grand talent au service duquel était attaché Teika ${ }^{3}$. Plutôt que de concours, il s'agit en réalité d'œuvres originales composées de poèmes choisis pour leur qualité et arrangés en un ensemble cohérent, à la manière d'une mosaïque. Il existe un 
manuscrit autographe de notre texte, conservé dans le fonds de la bibliothèque Hokuni 穂久雨文庫; il s'agit d'une copie que Teika fit après coup - en se fondant peut-être sur l'exemplaire de Yoshitsune - car un emprunteur indélicat avait égaré son manuscrit personnel. Lors de la copie, Teika opéra quelques corrections ; c'est une édition de ce texte révisé que nous utiliserons ici ${ }^{4}$.

2 Dans ces deux concours, Teika a placé les poèmes du Genji monogatari à gauche. Ce simple détail, a priori insignifiant, nous indique que dans son esprit, les poèmes du Genji étaient d'une qualité supérieure ${ }^{5}$. En effet, lors des utaawase, deux équipes s'affrontaient ; la place de gauche était réservée aux hôtes d'honneur. L'aspect le plus fascinant de nos concours est la manière dont les poèmes ont été appariés et recontextualisés. Lors des concours réels, les poèmes étaient le plus souvent composés sur des sujets imposés (dai) et traitaient le même thème. Dans le cas présent, les waka sont empruntés à des romans différents, leur appariement représente donc une gageure. En outre, s'agissant de poèmes extraits de romans, la connaissance du contexte narratif est absolument nécessaire à leur compréhension ; Teika a donc rédigé des notices introductives, kotobagaki, plus ou moins longues selon les cas, afin de resituer les poèmes. Pour comprendre de quelle manière Teika a procédé, nous concentrerons notre analyse sur les manches 45 à 52 du Hyakuban utaawase, le premier des deux concours. Les manches 45 à 47 closent la section des poèmes de " séparation »; les manches 48 à 52 ouvrent celle des poèmes de "voyage ». Dans les exemples que nous allons examiner, les poèmes de gauche sont - à une exception près extraits du chapitre "Suma », ceux de droite, appartiennent à différents chapitres du Sagoromo monogatari. Rappelons, avant d'entrer dans le vif du sujet, que le Genji monogatari contient un total de 795 waka; le chapitre "Suma ", avec 48 pièces, est celui dont la maille poétique est la plus dense $^{6}$ : ce chapitre est également le mieux représenté dans notre concours (Teika en a retenu 13 poèmes ${ }^{7}$ ). Le Sagoromo monogatari, qui est un roman beaucoup plus court - il représente en volume à peu près le quart du Genji - contient quant à lui 216 waka : cela signifie que près de la moitié des poèmes du roman figurent dans notre concours.

Examinons à présent la manche 45 , la première de notre corpus :

\begin{tabular}{|l|l|}
\hline \multicolumn{2}{|l|}{ Gauche : Séparation [lors du départ pour] Suma } \\
\hline Uki yo woba & De ce monde de tristesse \\
\hline Ima zo wakaruru & A présent je m'éloigne \\
\hline Todomaramu & Et ma réputation \\
\hline Na woba Tadasu no & Au dieu de Tadasu je la confie \\
\hline Kami ni makasete & Afin qu'il la restaure. \\
\hline
\end{tabular}

Dans le roman, ce poème ( $\mathrm{n}^{\circ}$ 181) est mis dans la bouche du Genji ${ }^{8}$. Ce dernier, qui doit s'exiler à Suma, décide, la nuit précédant son départ, d'aller se recueillir sur la tombe de son père, l'empereur retiré Kiritsubo. En chemin, il rend visite pour lui faire ses adieux à Fujitsubo, une épouse de son père qu'il a lui aussi aimée. Après leur entrevue, bouleversé, il reprend le chemin pour se rendre sur la tombe de son père et passe alors 
devant le Sanctuaire d'En-Bas de Kamo ; il s'y arrête pour annoncer à la divinité son départ. C'est alors qu'il compose le poème ${ }^{9}$. Dans le Genji monogatari, ce waka n'est donc pas un poème d'amour ; Teika invite toutefois le lecteur à lire en filigrane les adieux à Fujitsubo.

Regardons à présent le poème de droite :

\begin{tabular}{|c|c|}
\hline Namida nomi & Alors que mes larmes, \\
\hline Yodomanu kawa to & S'écoulent comme une rivière \\
\hline Nagaretsutsu & Dont le cours jamais ne stagne \\
\hline Wakare no michi wa & Le chemin de la séparation, lui, \\
\hline Yuki mo yararezu & Sera bien difficile à parcourir \\
\hline
\end{tabular}

Ce poème (chapitre $\left.3, n^{\circ} 184\right)^{10}$ est l'œuvre de Sagoromo, le héros du roman qui, accablé par des échecs successifs dans ses relations amoureuses, a décidé de se retirer du monde (shukke). La nuit précédant son départ, il rend discrètement visite à Genji no Miya, la Vestale de Kamo (Sai.in 斎院), qu'il aime depuis leur enfance passée ensemble. Il s'entretient galamment avec elle et, de retour chez lui à l'aube, discute tendrement avec sa fille, Wakamiya, la jeune princesse, qui n'est encore qu'une toute jeune enfant à qui il ne peut faire part de sa décision. L'idée de l'abandonner sans soutien rend la perspective du départ extrêmement pénible. C'est à ce moment qu'il compose son poème ${ }^{11}$. Contrairement à ce que nous dit la notice introductive, ce poème n'a pas été composé lors de la séparation d'avec la vestale (Sai.in), mais au moment où Sagoromo s'apprête à abandonner sa fille. Teika a donc sciemment modifié le contexte de composition de cette pièce afin d'accentuer la similarité entre les deux waka de cette manche. Dans notre concours, les deux poèmes sont présentés comme des pièces composées par le héros du roman qui fait ses adieux à une femme aimée au moment où, dans un contexte difficile, il décide de quitter le monde (pour l'exil ou pour entrer en religion); les deux pièces contiennent en outre un mot signifiant la séparation (wakaruru "se séparer », wakare "séparation »). La similitude est renforcée par le fait que le Genji se trouve dans le sanctuaire de Kamo et que la femme à qui, selon la notice introductive, Sagoromo fait ses adieux, n'est autre que la vestale de ce même sanctuaire.

Examinons la manche suivante :

\begin{tabular}{|l|l|}
\hline \multicolumn{2}{|l|}{ Manche 46} \\
\hline \multicolumn{2}{|l|}{ Gauche : Séparation [lors du départ pour] Suma } \\
\hline Murasaki no ue & \\
\hline Oshikaranu & En échange de cette vie \\
\hline
\end{tabular}




\begin{tabular}{|l|l|}
\hline Inochi ni kahete & A laquelle je n'ai plus goût \\
\hline Me no mae no & Je voudrais suspendre \\
\hline Wakare wo shibashi & Ne serait-ce qu'un instant \\
\hline Todome teshigana & Notre imminente séparation! \\
\hline
\end{tabular}

5 Le jour de son départ pour Suma, le Genji passe la journée avec Murasaki no ue, son grand amour. Ils s'entretiennent de choses et d'autres et, à la nuit tombée, comme le Genji se prépare à partir, il la voit si belle au clair de lune qu'un terrible sentiment d'inquiétude l'envahit à l'idée de l'abandonner sans soutien. Il compose alors un poème dans lequel il lui confesse la douleur de se séparer d'elle alors qu'il pensait vivre à ses côtés jusqu'au terme de sa vie. Notre waka $\left(\mathrm{n}^{\circ}{ }^{186}\right)$ est la réponse de Murasaki no ue au poème du Genji ${ }^{12}$. Examinons à présent le poème de droite :

\begin{tabular}{|l|l|}
$\begin{array}{l}\text { Droite : [Comme Sagoromo avait écrit dans son poème :] «Car tout comme la lune, je ne sais où } \\
\text { s'achèvera ma course }{ }^{13} \text {, elle répondit: }\end{array}$ \\
\hline La Vestale de Kamo & Devenez la lune \\
\hline Tsuki danimo & Et pourvu que les nuages \\
\hline Yoso no muragumo & Ne vous tiennent à l'écart \\
\hline Hedatezu ha & Nuit après nuit sur ma manche \\
\hline Yona yona sode ni & Votre reflet je contemplerai \\
\hline Utsushite mo mimu &
\end{tabular}

6 Ce poème (chapitre $4, n^{\circ} 188$ ) est mis dans la bouche de la Vestale de Kamo (Sai.in) alias Genji no Miya, déjà apparue à la manche précédente. Nous sommes toutefois à un autre moment du roman. Sagoromo, qui doit prochainement devenir empereur, lui rend visite. Son nouveau statut condamnant leurs relations, les deux amants décident d'un commun accord de se séparer. Sagoromo, très peiné, lui présente un poème dans lequel il exprime son inquiétude de ne savoir quand ils se reverront ${ }^{14}$. Le poème retenu dans notre manche est la réponse de la Vestale, qui reprend l'image de la lune présente dans la pièce de Sagoromo : elle lui confesse qu'elle continuera à penser à lui en observant le reflet de la lune venant se loger dans les larmes qu'elle versera sur son vêtement. Les deux poèmes de cette manche sont des pièces composées par des femmes contraintes par les circonstances de se séparer de l'homme qu'elles aiment.

7 La manche suivante, qui clôt la section « séparation », met en lice non plus un poème de «Suma » mais une pièce du chapitre «Akashi ». Nous allons toutefois l'examiner pour ne pas briser l'unité de notre corpus.

Manche 47 


\begin{tabular}{|l|l|}
\hline \multicolumn{2}{|l|}{ Gauche : Alors que le Genji s'apprêtait à rentrer à la capitale } \\
\hline La Dame d'Akashi & \\
\hline Toshi hetsuru & Cette cabane au toit de chaume \\
\hline Tomaya mo arete & Où des années j'ai vécu \\
\hline Uki nami no & Va se délabrer \\
\hline Kaheru kata ni ya & Et à ces vagues qui refluent vers vous \\
\hline Mi wo taguhemashi & Mon corps voudrais abandonner \\
\hline
\end{tabular}

8 Ce poème $\left(n^{\circ} 238\right)$ est composé par la dame d'Akashi - une maîtresse du héros, connue lors de son d'exil. Cette pièce est la réponse au waka que le Genji venu nuitamment lui rendre visite pour lui annoncer son retour imminent à la capitale, lui a fait tenir le lendemain matin, au moment de son départ. Dans son poème, le Genji s'inquiète de la laisser sans soutien ; la dame d'Akashi lui fait comprendre que son départ signifie pour elle la déchéance ${ }^{15}$.

Examinons à présent le poème de droite :

\begin{tabular}{|l|l|}
\hline \multicolumn{2}{|l|}{ Droite : Alors qu'elle devait prendre la décision de partir au loin } \\
\hline Princesse Asukai & \\
\hline Hanakatsumi & Fleurs de zizanie ; \\
\hline Katsu miru danimo & A peine vous ais-je rencontré \\
\hline Aru mono wo & Et déjà je languis, \\
\hline Asaka no numa ni & Le marais d'Asaka va-t-il manquer d'eau \\
\hline Mizu ya taenamu & Et nous, ne jamais plus nous revoir? \\
\hline
\end{tabular}

9 Ce poème (chapitre $1, n^{\circ} 23$ ) est mis dans la bouche de la princesse Asukai, l'une des conquêtes de Sagoromo. L'auteur joue dans cette pièce sur l'homophonie entre hanakatsumi une plante aquatique mal identifiée, et katsu mi « à peine vous ais-je vu » ${ }^{16}$. L'image de la plante aquatique appelle celle du marais d'Asaka et son jeu de mots sur mizu « l'eau » et « ne pas voir $»^{17}$. Asukai compose cette pièce lors d'une visite nocturne de Sagoromo. Ils échangent des serments d'amour, mais Asukai sait qu'elle est promise au fils de sa nourrice et que cette dernière doit l'emmener loin de la capitale : elle ignore donc s'ils pourront se revoir un jour ${ }^{18}$. Comme à la manche précédente, les deux pièces appariées ici sont adressées par des femmes amoureuses à leur amant qu'elles craignent de ne jamais revoir. La similitude de situation entre les deux pièces est renforcée par le recours commun à l'élément liquide (les vagues, nami, dans le premier et l'eau, mizu dans le second). 
10 Nous abordons à présent la section «voyage » de notre concours. Dans la littérature japonaise classique, le voyage n'est nullement considéré comme un divertissement ; il s'agit d'une expérience souvent douloureuse, impliquant solitude, séparation d'avec les êtres chers, et inquiétude face aux dangers éventuels.

Observons maintenant la manche 48 , la première de la section :

\begin{tabular}{|l|l|}
\hline \multicolumn{2}{|l|}{ Gauche : Dans la baie de Suma, alors qu'il voyait à proximité s'élever de la fumée } \\
\hline Yama gatsu no & Les rustres de la montagne \\
\hline Ihori ni takeru & Font brûler dans leurs cabanes \\
\hline Shiba shiba mo & De menues branches ; \\
\hline Koto tohi konamu & Qu'ils viennent plus souvent prendre de mes nouvelles \\
\hline Kohuru sato bito & Ceux dont je me languis, restés au pays! \\
\hline
\end{tabular}

11 Le Genji est désormais sur son lieu d'exil. Loin de la capitale, tout lui semble étrange, même la fumée des feux de brindilles que les habitants allument près de sa résidence. Ces feux sont l'occasion pour l'auteur de jouer dans son poème ( $\left.n^{\circ} 208\right)$ sur l'homophonie des termes shiba « broussailles ", " branches », et shibashiba « souvent » ${ }^{19}$. Voyons à présent le poème de droite :

\begin{tabular}{|l|l|}
\hline \multicolumn{2}{|l|}{ Droite : A l'aube où, accompagnée de sa nourrice, elle prenait la route } \\
\hline Asukai & \\
\hline Ama no to wo & Que la porte céleste \\
\hline Yasurahi ni koso & A l'aube, indécise \\
\hline Ideshika to & J'ai franchi \\
\hline Yuhutsuke-dori yo & Dis-le si on t'interroge \\
\hline Towaba kotaheyo & ô coq annonceur du jour! \\
\hline
\end{tabular}

12 Ce poème (chapitre $1, \mathrm{n}^{\circ} 34$ ) est composé par Asukai (déjà apparue à la manche précédente), alors que sa nourrice l'emmène contre son gré pour la conduire en Tsukushi et la donner à son fils ${ }^{20}$. L'expression "porte céleste » désigne ici métaphoriquement la porte de la résidence d'Asukai. La similitude entre les deux poèmes de cette manche - moins flagrante que dans les précédentes - réside, nous semble-t-il, dans l'utilisation du verbe tohu qui signifie à la fois «demander» et « rendre visite ».

Manche 49 


\begin{tabular}{|c|c|}
\hline \multicolumn{2}{|c|}{ L'ancien ministre des Affaires suprêmes [alias le Conseiller] } \\
\hline Akanaku ni & Contre leur gré \\
\hline Kari no tokoyo wo & Les oies sauvages quittent \\
\hline Tachi wakare & Le pays de l'immortalité / votre séjour provisoire : \\
\hline Hana no miyako ni & En route pour la capitale des fleurs \\
\hline Michi ya madowamu & Elles vont sans doute s'égarer. \\
\hline
\end{tabular}

13 Ce poème ( $\mathrm{n}^{\circ}$ 213) est mis dans la bouche du Conseiller. Ce dernier, un intime du Genji, est venu lui rendre une brève visite dans son exil. Un vol d'oies sauvages qui traverse le ciel à l'aube, alors qu'ils sont en train de discuter, est prétexte à un échange de poèmes. Dans sa pièce, le Conseiller chante la peine qu'il a de quitter le Genji et la difficulté qu'il aura à regagner la capitale tant l'émotion le trouble ${ }^{22}$. Examinons à présent le poème de droite :

\begin{tabular}{|l|l|}
$\begin{array}{l}\text { Droite : Comme il s'était rendu pour la première fois chez Ippon no Miya, à l'aube, dans le palais de } \\
\text { la Première Avenue, seul et perdu dans ses pensées [il composa] : }\end{array}$ \\
\hline Shirasebaya ${ }^{23}$ & Puissiez-vous connaître \\
\hline Tokoyo hanareshi & Les sanglots que versent \\
\hline Karigane no & Les oies sauvages qui \\
\hline Omohi no hoka ni & Loin du pays de l'immortalité \\
\hline Kohite naku ne wo & Se languissent de ce qu'elles aiment ! \\
\hline
\end{tabular}

14 Ce poème (chapitre $3, \mathrm{n}^{\circ} 98$ ) est composé par Sagoromo. Après une première nuit passée chez Ippon no Miya, une femme que la pression sociale l'a contraint à épouser alors qu'il n'existe aucun sentiment entre eux, il se rend, dépité, à l'aube, au palais de la Première avenue (Ichijō no miya). C'est là que réside Wakamiya, la fille qu'il a eue secrètement avec Onna ni no miya (la fille de l'empereur Saga-in), appelée Nyūdō no miya depuis son entrée en religion. Dans une grande confusion mentale, il pense alors à la mère de son enfant à qui il adresse ce poème où il lui confesse son amour ${ }^{24}$. L'appariement des poèmes de cette manche est dû à l'emploi dans les deux pièces de l'image des oies sauvages, kari/karigane et du mot tokoyo qui désigne le "pays des immortels", "pays des esprits" car, selon la tradition poétique, les oies sauvages étaient douées du pouvoir de se rendre dans l'au-delà et même de communiquer avec les esprits des défunts ${ }^{25}$. Dans le poème du Genji monogatari, l'expression kari no tokoyo, « les oies [quittent] le pays des immortels », nous permet de lire en filigrane kari no toko, "[votre] résidence provisoire ", résidence d'exil ; dans celui du Sagoromo monogatari, le 
"Pays de l'immortalité » sert simplement de métaphore pour exprimer la douleur de l'éloignement (de leur terre d'élection pour les oies ; de l'être aimé pour Sagoromo).

\begin{tabular}{|l|l|}
\hline \multicolumn{2}{|l|}{ Manche 50} \\
\hline \multicolumn{2}{|l|}{ Gauche : Dans la baie de Suma } \\
\hline Kohi wabite & Le bruit des vagues de la baie \\
\hline Nakune ni magahu & se confond avec les sanglots que je verse \\
\hline Uranami ha & Par langueur d'amour: \\
\hline Omohu kata yori & Serait-ce que le vent souffle \\
\hline Kaze ya fukuramu & Depuis le lieu où vivent celles que j'aime? \\
\hline
\end{tabular}

15 Ce poème ( $\mathrm{n}^{\circ}$ 199) est composé par le Genji qui, constatant la similitude entre le bruit des vagues et celui de ses pleurs, feint de supposer que le vent porte jusqu'à son lieu d'exil les sanglots que versent en pensant à lui les femmes qu'il aime, restées à la capitale ${ }^{26}$.

\begin{tabular}{|l|l|}
\hline \multicolumn{2}{|l|}{ Droite : Comme il se rendait sur le mont Kōya } \\
\hline Ukifune no & Sur cette barque ballottée par les flots \\
\hline Tayori ni yukamu & Je m'en vais lui rendre hommage : \\
\hline Watatsumi no & Dites-moi où elle repose \\
\hline Soko to woshiheyo & Au fond de ce vaste océan \\
\hline Ato no shiranami & Vagues blanches du sillage! \\
\hline
\end{tabular}

16 Ce poème (chapitre 2, $\mathrm{n}^{\circ} 76$ ) est mis dans la bouche de Sagoromo qui se rend au Mont Kōya en pèlerinage. Traversant en barque la rivière Yoshino, il repense à Asukai qui s'est jetée du bateau qui la conduisait en Tsukushi et qu'il croit morte noyée ${ }^{27}$. L'appariement de ces deux pièces repose à la fois sur le recours commun à l'image de la mer et des vagues mais aussi sur la situation : les deux poèmes ont été composés par les héros qui pensent avec douleur à une femme aimée dont ils sont séparés par le destin.

\begin{tabular}{|l|}
\hline \multicolumn{2}{|l|}{ Manche 51} \\
\hline Gauche : A Ise \\
\hline L'épouse de l'ancien prince impérial ${ }^{28}$ : \\
\hline Ise shima ya $\quad$ Sur l'île d'Ise \\
\hline
\end{tabular}




\begin{tabular}{|l|l|}
\hline Shihohi no kata ni & Quand bien même à marée basse \\
\hline Asarite mo & Des coquillages on chercherait \\
\hline Ihu kahi naki ha & Ce serait hélas en vain, \\
\hline Waga mi narikeri & Tout comme est vaine ma personne. \\
\hline
\end{tabular}

17 Ce poème ( $\mathrm{n}^{\circ}$ 195) est composé par Rokujō no Miyasundokoro, une princesse que le Genji a jadis aimée et qui réside pour lors à Ise. Apprenant que celui-ci se trouve en exil à Suma, elle lui fait porter une lettre dans laquelle figure notre waka. En évoquant la morosité de sa vie à Ise, elle espère toucher le Genji et renouer ainsi une relation qui avait été interrompue ${ }^{29}$.

\begin{tabular}{|l|l|}
\hline \multicolumn{2}{|l|}{ Droite : Dans le bateau } \\
\hline Asukai & \\
\hline Nagarete mo & Si les flots m'emportent \\
\hline Ahuse ariya to & Nous reverrons-nous jamais? \\
\hline Mi wo nagete & Je me jette à l'eau \\
\hline Mushiake no seto ni & Dans le chenal de Mushiake \\
\hline Machi kokoromimu & Et verrai bien ce qu'il adviendra! \\
\hline
\end{tabular}

18 Le poème de droite (chapitre $1, \mathrm{n}^{\circ} 42$ ) est composé par Asukai qui, emmenée en Tsukushi par le fils de sa nourrice et ne pouvant survivre à la séparation d'avec Sagoromo, décide au cours de la traversée de se jeter à l'eau ${ }^{30}$. L'appariement de ces deux pièces repose essentiellement sur la similitude de situation : il s'agit de deux waka composés par des femmes et adressés à l'homme qu'elles aiment dont elles ne savent pas si elles pourront le revoir un jour. Cette similitude entre les deux pièces est renforcée par le recours à l'image de la mer, ainsi que par l'utilisation du terme mi / wagami que l'on peut traduire, selon les cas, par «mon corps ", "ma personne ", «moi ».

Examinons à présent la dernière manche de notre corpus :

\section{Manche 52}

Gauche : Dans la baie de Suma, au moment où l'on célébrait les lustrations du jour du Serpent, comme il contemplait la surface de la mer qui s'étendait à l'infini, lumineuse et calme, il vit qu'on fabriquait des mannequins de bonne taille que l'on disposa sur une barque et qu'on lança à la mer ; [il composa] alors :

\begin{tabular}{|l|l}
\hline Shirazarishi & Entraîné par les courants,
\end{tabular}




\begin{tabular}{|l|l|}
\hline Ohoumi no hara ni & De cette mer jusqu'alors inconnue \\
\hline Nagare kite & tel un mannequin \\
\hline Hitokata ni ya ha & Ici j'ai échoué ; dès lors ma tristesse \\
\hline Mono ha kanashiki & Pourrait-elle être ordinaire $?^{31}$ \\
\hline
\end{tabular}

19 La vision des mannequins est pour le Genji l'occasion de composer un poème ( $\left.\mathrm{n}^{\circ} 215\right)$ dans lequel il s'interroge sur le cours de son destin et son infortune ${ }^{32}$. Observons à présent le poème de droite :

\begin{tabular}{|l|l|}
\hline \multicolumn{2}{|l|}{ Droite : Se souvenant d'Asukai } \\
\hline Karadomari & Telle une épave \\
\hline Soko no mikuzu to & Elle s'est échouée au fond des eaux - \\
\hline Nagareshi wo & A Karadomari ; parmi les vagues \\
\hline Seze no iwanami & Qui dans les bas-fonds, battent les rochers \\
\hline Tazune teshigana & A sa recherche, je voudrais aller! \\
\hline
\end{tabular}

20 Le poème de droite (chapitre $2, \mathrm{n}^{\circ}$ 66) est composé par Sagoromo qui, apprenant qu'Asukai s'est précipitée dans les flots, souhaite se rendre sur les lieux de son suicide ${ }^{33}$.

21 L'appariement de ces deux pièces repose essentiellement sur l'emploi du verbe nagaru qui signifie dans nos poèmes « dériver au gré du courant » et illustre bien la souffrance de l'homme aux prises avec un destin auquel il ne peut s'opposer. On peut également noter un parallèle de situation : ces deux pièces sont des cris du cœur poussés par des héros que la souffrance accable.

22 Après avoir examiné chacune des manches, il ressort clairement que trois éléments président à l'appariement des poèmes : 1) la similitude entre les personnages ; 2) une similitude du contexte de production, de la situation qui a suscité le poème et donc du thème chanté ; 3 ) une similitude dans l'expression (ces trois éléments pouvant être combinés) $)^{34}$. Le fait que le Sagoromo monogatari soit très influencé par le Genji monogatari, et que l'on trouve dans les deux romans nombre de situations similaires, a sans doute favorisé la qualité de l'appariement.

23 Il convient à présent de s'interroger sur la structure d'ensemble de notre corpus. Nous remarquons tout d'abord que Teika n'a pas retenu l'ordre des poèmes tel qu'il apparaît dans les romans ${ }^{35}$. En outre, le fait que la plupart des poèmes du Genji monogatari de notre corpus ont été pris au chapitre «Suma » alors que ceux du Sagoromo monogatari qui leur sont appariés appartiennent aux quatre chapitres du roman, nous indique que Teika a, dans un premier temps, choisi les poèmes du Genji et les a ensuite appariés à des poèmes du Sagoromo ${ }^{36}$. Contrairement à ce que l'on peut observer dans les anthologies impériales (chokusenshū 勅撰集), on ne relève pas de réelle progression au sein d'une section. Teika a conçu chaque manche comme une unité indépendante, 
ménageant toutefois une certaine continuité d'une manche sur l'autre ${ }^{37}$. Ainsi, le poème de Murasaki no ue à la manche 46 (la deuxième de notre corpus), dans lequel elle dit souhaiter retarder le départ $\mathrm{du}$ Genji, peut-il être lu comme la réponse au poème du Genji de la manche 45 (la première) dans lequel il fait ses adieux avant de partir en exil. La manche 47, qui clôt la section "séparation" est indépendante. Au début de la section « voyage » (manche 48), nous voyons le Genji demander dans son poème que ses amis lui rendent visite ; à la manche 49, il est exaucé : le Conseiller vient le voir. A la manche 50, le Genji croit entendre dans le bruit des vagues les sanglots de femmes qui se languissent de lui et, effectivement, à la manche 51, c'est Rokujō no Miyasundokoro qui lui envoie un poème lui signifiant qu'elle désire le revoir. Enfin, le poème de la manche 52 (la dernière de notre corpus), dans lequel le Genji exprime la tristesse de son destin en évoquant les mannequins flottant sur la mer au gré des flots, trouve un écho dans le poème de la manche 53 , une pièce de Murasaki no ue dans laquelle cette dernière évoque son chagrin et son inquiétude en recourant à l'image des « vagues » de larmes qu'elle verse ${ }^{38}$. Cette continuité n'est qu'exceptionnellement annoncée par les notices introductives (kotobagaki) ${ }^{39}$. Dans notre corpus, la fonction essentielle de ces notices est de rappeler les circonstances de composition - il s'agit là de la fonction "classique» des kotobagaki telle qu'on peut l'observer, par exemple, dans les anthologies impériales. S'agissant de poèmes extraits de romans, leur fonction est également de rappeler le contexte narratif ${ }^{40}$. Nous avons vu que Teika n'a pas hésité, à l'occasion, à modifier dans ses notices le contexte de composition; il s'agissait là de renforcer la cohérence au sein d'une même manche ${ }^{41}$.

Il convient à présent, pour clore cette étude, de s'interroger sur la finalité de l'entreprise : pourquoi apparier des poèmes extraits de romans? Si l'on ne peut exclure une visée didactique - enseigner quels sont les meilleurs poèmes extraits de romans - il nous semble plus juste de chercher la réponse dans l'histoire du waka. En effet, la compilation de notre concours s'inscrit dans une époque - et dans un mouvement - qui a scellé la reconnaissance de l'utilisation de poèmes et de passages en prose extraits de monogatari comme matériaux pouvant être employés dans de nouvelles compositions ; Fujiwara no Shunzei (1114-1204), le père de Teika, a d'ailleurs joué un rôle décisif dans cette évolution ${ }^{42}$. Le waka s'est, tout au long de son histoire, nourri - consciemment ou inconsciemment - de références intertextuelles. À l'époque de notre concours, la pratique appelée honkadori 本歌取 "prendre un poème de base", qui consiste à composer un nouveau poème en empruntant des éléments reconnaissables à un waka ancien, connaissait une vogue exceptionnelle ${ }^{43}$. Il s'agissait par ce mode de composition d'enrichir le sens du nouveau waka en évoquant en filigrane la pièce utilisée comme poème de base. Lorsqu'on se souvient que notre concours fut commandé par Fujiwara no Yoshitsune, qui s'est toujours attaché à exploiter de nouvelles pistes pour renouveler, enrichir et développer la composition du waka, il devient alors certain que notre concours s'inscrit dans une même recherche visant à créer de nouvelles sensations poétiques. En empruntant et en appariant les poèmes de deux romans choisis parmi les chefs-d'œuvre de la littérature et connus de tous, en écrivant les notices, Teika n'exprimait pas seulement son admiration pour ces monogatari; il mettait en œuvre une subtile mécanique destinée à livrer l'un après l'autre tous les effets qu'autorise le waka: provoquer la surprise, l'émotion, susciter dans l'esprit du lecteur de nouvelles associations, de nouvelles sensations. En d'autres termes, il s'agissait, par l'agencement des poèmes, par leur remise en contexte, de donner naissance à de nouveaux mondes ${ }^{44}$. 


\section{BIBLIOGRAPHIE}

\section{Éditions des sources}

Monogatari nihyakuban utaawase, in Higuchi Yoshimaro (éd.), Ōchō monogatari shūkasen, vol. 1, coll. « Iwanami Bunko », Tōkyō, Iwanami shoten, 1987.

Genji monogatari, Ishida Jōji et Shimizu Yoshiko (éd.), coll. « Shinchō Nihon koten shūsei », Tōkyō, Shinchōsha, 1976-1985, 8 tomes.

Kunchū Meigetsuki, Inamura Eiichi (éd.), Matsue, Matsue Imai shoten, 2002, réed. 2003, 8 tomes.

Sagoromo monogatari, Suzuki Kazuo (éd.), coll. « Shinchō Nihon koten shūsei », Tōkyō, Shinchōsha, 1985-1986, 2 tomes.

Kyōgoku chūnagon sōgo, in Hisamatsu Sen.ichi (éd.), Karonshū 1, coll. « Chūsei no bungaku », Tōkyō, Miyai shoten, 1971, p. 333-338.

Sieffert, René (trad.), Le Dit du Genji, Cergy, POF, 1988.

Études

Fujihira Haruo, « Shinkokinshū to Genji monogatari, Teika no honkadori to Genji monogatari no hiki uta », in Fujihira Haruo chosakushū, vol. 5, Tōkyō, Kasama shoin, 2003.

Hérail, Francine, Fonctions et fonctionnaires japonais au début du XIe siècle, 2 tomes, Paris, POF, 1977.

Higuchi Yoshimaro, « Genji monogatari to monogatari shūkasen », in Takahashi Tōru et Kubo Tomotaka (éd.), Shinkō Genji monogatari o manabu hito no tameni, Kyōto, Sekai shisōsha, 1995.

Higuchi Yoshimaro, « Genji Sagoromo hyakuban utaawase kō, burui, hairetsu o chūshin ni », Aichi daigaku kokubungaku, n 12, 1971, repris dans Higuchi Yoshimaro, Heian Kamakura jidai san.itsu monogatari no kenkyū, Tōkyō, Hitaku shobō, 1982.

Higuchi Yoshimaro, « Genji Sagoromo hyakuban utaawase no hairetsu ni tsuite », Bungaku Gogaku, $\mathrm{n}^{\circ}$ 57, 1982, repris dans Higuchi Yoshimaro, Heian Kamakura jidai san.itsu monogatari no kenkyū, Tōkyō, Hitaku shobō, 1982.

Higuchi Yoshimaro, « Matsura no miya monogatari, Monogatari nihyakuban utaawase no seiritsu jiki ni tsuite », Kokugo to Kokubungaku vol. 57, n 5, 1980, repris dans Higuchi Yoshimaro, Heian Kamakura jidai san.itsu monogatari no kenkyū, Tōkyō, Hitaku shobō, 1982.

Higuchi Yoshimaro, « Monogatari gohyakuban utaawase hairetsu kō », Aichi kyōiku daigaku kenkyū hōkoku, n 33, 1974.

Higuchi Yoshimaro, « Monogatari utaawase to monogatari kashū », in Waka to monogatari, coll. «Waka bungaku ronshū » nº 3, Tōkyō, Kazama shobō, 1992.

Higuchi Yoshimaro, « Monogatari nihyakuban utaawase to Mumyōzōshi », Kokugo kokubungaku hō, $\mathrm{n}^{\circ} 38,1981$.

Ii Haruki, « Monogatari nihyakuban utaawase no honbun », Gobun, n 48, 1987.

Komachiya Teruhiko, Ōchō bungaku no utakotoba hyōgen, Tōkyō, Wakakusa shobō, 1997.

Kubota Jun, «Genji monogatari to Fujiwara no Teika, Chikatada no musume oyobi sono shūhen », in Fujiwara no Teika to sono jidai, Tōkyō, Iwanami shoten, 1994. 
Ōtsuki Osamu, « Genji, Torikaebaya no tsugai ni tsuite - Monogatari gohyakuban utaawase no hairetsu kara », Gobun, nº 48, 1987.

Ōtsuki Osamu, « Genji, Yoru no nezame no tsugai ni tsuite - Monogatari gohyakuban utaawase no hairetsu kara », Kōnan kokubun, n 33, 1986 (première partie) ; Kōnan joshi daigaku kenkyū kiyō, $\mathrm{n}^{\circ}$ 22, 1986 (deuxième partie).

Pigeot, Jacqueline, Questions de poétique japonaise, Paris, PUF, 1997.

Tabuchi Kumiko, « Monogatari nihyakuban utaawase no hōhō », in Genji monogatari kenkyū, nº 9, 2004.

Tabuchi Kumiko, « Monogatari nihyakuban utaawase no seiritsu to kōzō », in Kokugo to kokubungaku, numéro spécial, mai 2004.

Takada Hirohiko, Genji monogatari no bungaku shi, Tōkyō, Tōkyō daigaku shuppankai, 2003.

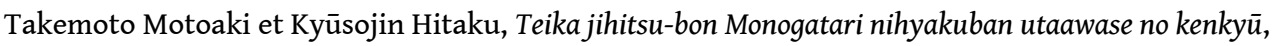
coll. « Mikan kokubun shiryō », vol. 1-1, Toyohashi, Mikan kokubun Shiryō kankōkai, 1955.

Terada Sumie, « La prose dans le contexte de la poésie, Le Roman du Genji et l'évolution poétique », in Éloge des sources, Arles, Philippe Picquier, 2004.

Teramoto Naohiko, Genji monogatari juyōshi ronkō, seihen, Tōkyō, Kazama shobō, 1970.

Tōno Yasuko, « Genji Sagoromo utaawase no tsugai to sono keisei », Mozu kokubun, nº 9, 1989.

Tōno Yasuko, « Monogatari nihyakuban utaawase no Genji uta to Teika », in Katagiri Yōichi (éd.), Ōchō no bungaku to sono keifu, Ōsaka, Izumi shoin, 1991.

Ueno Eiji, « Genji monogatari no kyōju to honbun, Monogatari nihyakuban utaawase shoshūbon o megutte », Kokugo kokubun, vol. 53, nº 1, 1984.

Vieillard-Baron Michel, Fujiwara no Teika et la notion d'excellence en poésie, théorie et pratique de la composition dans le Japon classique, Paris, Collège de France, Institut des Hautes Études Japonaises, 2001.

Yoneda Akemi, « Monogatari nihyakuban utaawase no kotobagaki ikkō », in Higuchi Yoshimaro (éd.), Ōchō waka to shiteki tenkai, Tōkyō, Kasama shoin, 1997.

\section{NOTES}

1. Dans son article « Monogatari nihyakuban utaawase no hōhō ", in Genji monogatari kenkyū n ${ }^{\circ} 9$ (2004), Tabuchi Kumiko souligne le soin que Teika porta à l'établissement et à la rationalisation des noms des personnages du Genji (désignés de multiples façons dans ce roman qui se déroule sur trois quarts de siècle) allant jusqu'à joindre à son concours un index des personnages-auteurs classés par ordre décroissant de rang (d'abord les hommes, puis les femmes) avec, pour chacun, le nombre de pièces retenues. Il s'agissait pour Teika, souligne Tabuchi, de conférer à son œuvre le caractère officiel des véritables concours et des anthologies impériales.

2. Le Sagoromo monogatari décrit les déboires sentimentaux du héros Sagoromo, qui, malheureux en amour, connaîtra cependant une carrière brillante, couronnée par son accès à la fonction suprême d'empereur. Ce roman, très influencé par le Genji monogatari, fut écrit avant 1086 ; il est attribué à Senji 宣旨, la "Dame chargée des ordres impériaux", qui était au service de la princesse impériale Baishi (1039-1096), une femme passionnée de littérature. Comme le prouve la citation des notes journalières de Fujiwara no Teika placée en exergue, le Sagoromo monogatari était, de par la qualité de ses poèmes, considéré comme le meilleur roman, après le Genji. 
3. En opérant une analyse minutieuse des différentes données historiques dont on dispose, Higuchi Yoshimaro réussit à situer la compilation de nos concours entre l'automne de l'année Kenkyū 4 (1193) et l'hiver de l'année Kenkyū 7 (1196) ; le concours de poèmes extraits de romans que l'empereur Gotoba demanda à Teika et à Fujiwara no Ariie 藤原有家 (1155-1216) de compiler en 1205 (cf. Meigetsuki 明月記, Genkyū 2 (1205), $12^{\mathrm{e}}$ mois, $7^{\mathrm{e}}$ jour) est de toute évidence un autre texte. Voir Higuchi Yoshimaro, « Matsura no miya monogatari, Monogatari nihyakuban utaawase no seiritsu jiki ni tsuite", Kokugo to Kokubungaku vol.57, ${ }^{\circ} 5$, 1980, repris dans: Higuchi Yoshimaro, Heian Kamakura jidai san.itsu monogatari no kenkyū, Tōkyō, Hitaku shobō, 1982, en particulier p. 23 et, du même auteur : «Genji monogatari to monogatari shūkasen », in Takahashi Tōru et Kubo Tomotaka (éd.), Shinkō Genji monogatari o manabu hito no tame ni, Kyōto, Sekaishisōsha, 1995, p. 254.

4. On trouvera un relevé des principales différences entre les deux versions de notre concours dans Higuchi Yoshimaro : "Monogatari utaawase to monogatari kashū », in Waka to monogatari, coll. « Waka bungaku ronshū » $n^{\circ}$ 3, Tōkyō, Kazama shobō, 1992, p. 64. Pour ce qui est de l'édition utilisée, voir la bibliographie en fin d'article.

5. Dans un texte intitulé Kyōgoku chunagon sogo 京極中納言相語 《Entretiens avec le second conseiller Kyōgoku [alias Teika]», rapportés par son disciple Fujiwara no Nagatsuna 藤原長綱, nous pouvons lire (p. 335) : «Lorsqu'on observe le style de Murasaki Shikibu, on s'aperçoit que le sens est exprimé de manière limpide, et que les poèmes sont composés élégamment, tant du point de vue de la forme générale [sugata] que de l'expression [kotoba] ». Le Genji monogatari est l'œuvre narrative à laquelle Teika fait le plus souvent référence dans sa poésie personnelle. Voir à ce sujet Sumie Terada, «La prose dans le contexte de la poésie, Le roman du Genji et l'évolution poétique », in Éloge des sources, reflets du Japon ancien et moderne, Arles, Philippe Picquier, 2004, p. 150-155, mais également Kubota Jun, « Genji monogatari to Fujiwara no Teika, Chikatada no musume oyobi sono shūhen », in Fujiwara no Teika to sono jidai, Tōkyō, Iwanami shoten, 1994, p. 289-328, et Fujihira Haruo, «Shinkokinshū to Genji monogatari, Teika no honkadori to Genji monogatari no hiki uta ", in Fujihira Haruo chosakushū, vol. 5, Tōkyō, Kasama shoin, 2003, p. 161-177.

6. Sur les multiples références poétiques qui émaillent le chapitre «Suma», voir Takada Hirohiko : « In.yō no sōzōsei - Suma no maki no hōhō », in Genji monogatari no bungaku shi, Tōkyō, Tōkyō daigaku shuppankai, 2003, p. 210-234.

7. Teika en a retenu 10 autres dans son second concours.

8. La numérotation des poèmes - ordonnés selon leur place dans le roman - reprend celle du Shinpen kokka taikan, vol. 5, Tōkyō, Kadokawa shoten, 1987.

9. Genji monogatari, vol. 2, p. 220 ; Le Dit du Genji, vol. 1, p. 257.

10. Pour le Sagoromo monogatari, nous reprenons également la numérotation du Shinpen kokka taikan, vol. 5, Tōkyō, Kadokawa shoten, 1987.

11. Sagoromo monogatari, vol. 2, p. 184.

12. Genji monogatari, vol. 2, p. 224 ; Le Dit du Genji, vol. 1, p. 259.

13. Sagoromo monogatari, vol. 2, p. 316.

Meguri awamu Nous reverrons-nous?

Kagiri dani naki Pour cette séparation

Wakare kana Il n'est même pas de terme,

Sora yuku tsuki no Car tout comme la lune dans le ciel

Hate wo shiraneba Je ne sais où s'achèvera ma course.

14. Ce poème, cité dans la note précédente, apparaît à la manche 1 de notre concours.

15. Genji monogatari, vol. 2, p. 300 ; Le Dit du Genji, vol. 1, p. 301.

16. Sur ce sens particulier voir le poème $n^{\circ} 677$ du Kokin wakashū.

17. Ces jeux de mots bien attestés figurent également dans le poème $n^{\circ} 677$ du Kokin wakashū.

18. Sagoromo monogatari, vol. 1, p. 76-77. 
19. Genji monogatari, vol. 2, p. 245 ; Le Dit du Genji, vol. 1, p. 272.

20. Sagoromo monogatari, vol. 1, p. 104-105.

21. René Sieffert traduit "conseiller», le terme saishō que Francine Hérail (Fonctions et fonctionnaires japonais au début du XI ${ }^{e}$ siècle, vol. 2, p. 110) rend par « auditeur »; nous conservons la traduction de R. Sieffert pour que le lecteur intéressé puisse se reporter à sa traduction du roman.

22. Genji monogatari, vol. 2, p. 252-253 ; Le Dit du Genji, vol. 1, p. 276.

23. Signalons que dans notre édition de référence du Sagoromo monogatari (vol.2, p. 92), le premier vers de ce poème est légèrement différent : (Kikasebaya : « Puissiez-vous entendre »).

24. Sagoromo monogatari, vol. 2, p. 91-92.

25. Voir l'article kari dans, Kubota Jun et Baba Akiko (éd.), Utakotoba utamakura daijiten, Tōkyō, Kadokawa shoten, 1999, p. 265-266.

26. Genji monogatari, vol. 2, p. 237 ; Le Dit du Genji, vol. 1, p. 267.

27. Sagoromo monogatari, vol. 1, p. 248-249.

28. Rokujō no Miyasundokoro a en effet été mariée dans sa jeunesse à un prince impérial; ce dernier était mort bien avant que commence sa liaison avec le Genji.

29. Genji monogatari, vol. 2, p. 232-233 ; Le Dit du Genji, vol. 1, p. 264.

30. Sagoromo monogatari, vol. 1, p. 121.

31. L'auteur joue dans cette pièce sur les deux sens de hitokata: «mannequin / simulacre » et " ordinaire».

32. Genji monogatari, vol. 2, p. 254 ; Le Dit du Genji, vol. 1, p. 277.

33. Sagoromo monogatari, vol. 1, p. 211.

34. Voir Teramoto Naohiko, Genji monogatari juyōshiron kō, seihen, Tōkyō, Kazama shobō, 1970, p. 300-301.

35. Dans notre corpus, les poèmes du Genji monogatari sont, par ordre d'apparition : 181, 186, 238 , 208, 213 199, 195, 215 ; ceux du Sagoromo monogatari : 142, 188, 23, 34, 98, 76, 42, 66.

36. Cette hypothèse est soutenue par Higuchi Yoshimaro, Heian Kamakura jidai san.itsu monogatari no kenkyū, Tōkyō, Hitaku shobō, 1982, p. 387.

37. Sur ce point, voir par exemple Higuchi Yoshimaro "Monogatari hyakuban utaawase no hairetsu " in Heian Kamakura jidai san.itsu monogatari no kenkyū, Tōkyō, Hitaku shobō, 1982, p. 371-386 et également, en français, Sumie Terada, art. cit., p. 160. Tabuchi Kumiko (« Monogatari nihyakuban utaawase no seiritsu to kōzō ", in Kokugo to kokubungaku, numéro spécial, mai 2004, p. 43) voit dans cette façon de placer les poèmes visant à ménager une continuité, l'influence des jika awase « concours de ses propres poèmes " pour lesquels un poète appariait à la manière d'un concours des pièces choisies dans sa production personnelle. Cette pratique connaissait une grande vogue à l'époque où fut compilé notre concours.

38. Genji monogatari, vol. 2, p. 260 ; Le Dit du Genji, vol. 1, p. 280.

39. Dans notre corpus, seules les notices introduisant les poèmes du Genji monogatari des manches 45 et 46 , identiques, indiquent une unité de temps et laissent entendre une éventuelle continuité, confirmée par les poèmes.

40. Sumie Terada (op. cit., p. 160-166) a défini trois catégories de notices introductives dans notre concours : la première assume une fonction déictique en nous donnant l'impression « d'être là, à ce moment-là ». Dans les notices de cette catégorie, c'est un fait sensible que l'on rappelle plutôt qu'un élément narratif explicite; elle cite pour illustrer cette catégorie la notice de la manche 48 : "Dans la baie de Suma, alors qu'il voyait à proximité s'élever de la fumée ». Les notices de la seconde catégorie, qui peuvent être très brèves, jouent un rôle narratif et résument la situation dans laquelle s'insère la composition (c'est le type de notice le plus largement représenté dans notre corpus); les notices de la troisième catégorie, plus exceptionnelles et parfois longues, s'apparentent à des citations remaniées. Pour les écrire, Teika emprunte différentes expressions à la prose du roman. L'exemple le plus éloquent est la notice de la 
manche 52 : «dans la baie de Suma, au moment où l'on célébrait les lustrations du jour du Serpent...»

41. Tabuchi Kumiko fait remarquer en outre ("Monogatari nihyakuban utaawase no seiritsu to kōzō», op. cit., p. 46) que Teika a gommé de ses notices tout élément pouvant porter atteinte à l'honneur de la famille impériale. Ainsi, à la manche 1 du concours (non citée), figure un poème dans lequel le Genji chante son désir de rencontrer Fujitsubo, l'épouse de son père (l'empereur), dont il est amoureux. Le nom de la femme et le caractère adultérin de cette relation est entièrement absent de la notice (mais demeure évident pour tout lecteur, familier du roman). Il s'agissait par ces censures de préserver le caractère officiel du concours.

42. On trouvera un rappel de l'histoire de cette reconnaissance du monogatari comme matériau pouvant être utilisé pour la composition de waka (et du rôle décisif de Shunzei) dans Sumie Terada, op. cit., p. 141-145, http://cipango.revues.org/597.

43. Sur le honkadori, voir Jacqueline Pigeot, Questions de poétique japonaise, p.52-55 et Michel Vieillard-Baron, Fujiwara no Teika et la notion d'excellence en poésie, p. 251-282; il convient de signaler qu'il existait également une pratique, moins fréquente, appelée honzetsu 本説, qui consistait à faire référence dans son waka à une œuvre en prose ou à un poème chinois.

44. Je tiens à remercier ici le Kokubungaku kenkyū shiryōkan (Institut national de la Littérature japonaise) qui m'a donné la possibilité de rassembler au Japon la bibliographie nécessaire à la rédaction de cet article.

\section{RÉSUMÉS}

Cuvre du XIII ${ }^{\mathrm{e}}$ siècle qui présente l'appariement de poèmes extraits du Genji monogatari avec des pièces d'une œuvre qu'elle a inspiré, le Sagoromo monogatari, à la manière des concours de poèmes (utaawase).

A 13th century work comprised of poems from the Genji monogatari and a work which inspired it: the Sagoromo monogatari. Poems are presented side-by-side, in the style of traditional Japanese poetry competitions (utaawase).

\section{INDEX}

Keywords : Fujiwara no Teika (1162-1241), Genji monogatari, Heian, Literature, monogatari, Novel, Sagoromo monogatari, Tale of Genji, utaawase, Murasaki Shikibu (v. 973-v. 1014 or 1025)

Mots-clés : Dit du Genji, écriture, Fujiwara no Teika (1162-1241), Genji monogatari, monogatari, Murasaki Shikibu (v. 973-v. 1014 ou 1025), poésie, roman, Sagoromo monogatari, utaawase, waka Index géographique : Suma キーワード : Genji monogatari 源氏物語, monogatari 物語, Murasaki Shikibu 紫式部 (v. 973-v. 1014 ou 1025), Fujiwara no Teika 藤原定家 (1162-1241), shi 詩, shōsetsu 小説, Sagoromo monogatari 狭衣物語, utaawase 歌合, Suma 須磨, Heian jidai 平安時代 （794-1185), bungaku 文 学

Thèmes : littérature

Index chronologique : Heian 